\title{
The role of metabolism in bacterial persistence
}

\section{Stephanie M. Amato ${ }^{1+}$, Christopher H. Fazen ${ }^{1+}$, Theresa C. Henry ${ }^{2,3+}$, Wendy W. K. Mok ${ }^{1+}$, Mehmet A. Orman ${ }^{1 \dagger}$, Elizabeth L. Sandvik ${ }^{1+}$, Katherine G. Volzing ${ }^{1+}$ and Mark P. Brynildsen ${ }^{1,2 *}$}

\author{
${ }^{1}$ Department of Chemical and Biological Engineering, Princeton University, Princeton, NJ, USA \\ ${ }^{2}$ Department of Molecular Biology, Princeton University, Princeton, NJ, USA \\ ${ }^{3}$ Rutgers Robert Wood Johnson Medical School, Rutgers University, Piscataway, NJ, USA
}

\section{Edited by:}

Sebastien P. Faucher, McGill

University, Canada

Reviewed by:

Martin I. Voskuil, University of

Colorado Denver, USA

Jill Zeilstra-Ryalls, Bowling Green

State University, USA

Ray A. Larsen, Bowling Green State

University, USA

*Correspondence:

Mark P. Brynildsen, Hoyt Laboratory,

Department of Chemical and

Biological Engineering, Princeton

University, William Street,

Princeton, NJ 08544, USA

e-mail: mbrynild@princeton.edu

${ }^{+}$These authors have contributed

equally to this work and are listed in

alphabetical order.
Bacterial persisters are phenotypic variants with extraordinary tolerances toward antibiotics. Persister survival has been attributed to inhibition of essential cell functions during antibiotic stress, followed by reversal of the process and resumption of growth upon removal of the antibiotic. Metabolism plays a critical role in this process, since it participates in the entry, maintenance, and exit from the persister phenotype. Here, we review the experimental evidence that demonstrates the importance of metabolism to persistence, highlight the successes and potential of targeting metabolism in the search for anti-persister therapies, and discuss the current methods and challenges to understand persister physiology.

Keywords: bacterial persistence, metabolism, antibiotic tolerance, ppGpp, nutrient environment

\section{INTRODUCTION}

Bacterial cultures contain a small subpopulation of cells that cannot readily be killed by antibiotics (Bigger, 1944). These cells have been named persisters, and their existence can be detected from antibiotic kill curves, where the first, rapid killing regime represents the death of normal cells and the second, slower killing regime indicates the presence of persisters (Balaban et al., 2004; Kint et al., 2012). Further, when these survivors are cultured, they produce populations with antibiotic sensitivities identical to those of the original culture. This establishes persistence as a phenotypic trait, unique from antibiotic resistance where genetic determinants allow growth at higher antibiotic concentrations. Persisters are an important health concern because they are enriched in biofilms and thought to underlie the proclivity of biofilm infections to relapse following the conclusion of antibiotic therapy (Lewis, 2008, 2010). Persisters have proven to be difficult to analyze due to their transient nature, low abundance, and similarity to the viable but non-culturable (VBNC) cell-type (Roostalu et al., 2008; Orman and Brynildsen, 2013b). However, strong evidence, in the form of genetic- and microscopy-based data (Balaban et al., 2004; Lewis, 2010; Maisonneuve et al., 2013), exists to support that, while under antibiotic stress, persister tolerances are derived from inactivity of essential cell functions. While this is not always the case, as demonstrated in a study of isoniazid (a prodrug requiring activation) (Wakamoto et al., 2013), and dormancy is not essential for persistence prior to antibiotic stress (Orman and Brynildsen, 2013a), prolonged survival to the majority of antibiotics, in the absence of genetic mutations, requires inactivity of the antibiotic's primary target. To achieve and maintain this state, as well as reverse the process to repopulate environments, coordinated metabolic action is needed. Namely, metabolism would participate in cessation of essential functions, be needed to maintain culturability (e.g., sustain a minimal adenylate charge: $[\mathrm{ATP}+0.5 \mathrm{ADP}] /[\mathrm{ATP}+$ ADP + AMP] (Chapman et al., 1971), repair/resynthesize damaged proteins (Nystrom and Gustavsson, 1998)), and reactivate the cell during reawakening (Figure 1). We refer to this process as the persister metabolic program and summarize the accumulated evidence substantiating the importance of metabolism to the persister phenotype as well as current methods and challenges to studying the metabolism of these rare and transient phenotypic variants.

\section{GENOMIC STUDIES IDENTIFY METABOLIC GENES AS IMPORTANT TO THE PERSISTER PHENOTYPE}

Perturbations to genes that encode enzymes or regulators of metabolism have frequently been found to alter persister levels (Table 1). In one of the initial genomic screens for persistence, a library was generated through digestion of the Escherichia coli chromosome, ligation of the fragments into plasmids, and transformation of the library into E. coli (Spoering et al., 2006). Upon successive rounds of ampicillin (AMP) treatment and culturing of survivors, a plasmid carrying $g l p D$, encoding G3P-dehydrogenase that converts glycerol-3-phosphate (G3P) to dihydroxyacetonephosphate (DHAP), was found to increase the abundance of persisters. Further analysis identified additional enzymes in G3P metabolism important for persistence to AMP, ofloxacin (OFL), and ciprofloxacin (CIP) (Table 1). The importance of G3P to E. coli persistence was further supported by a transposon mutant screen where $g l p D$ mutants were found to increase persistence 


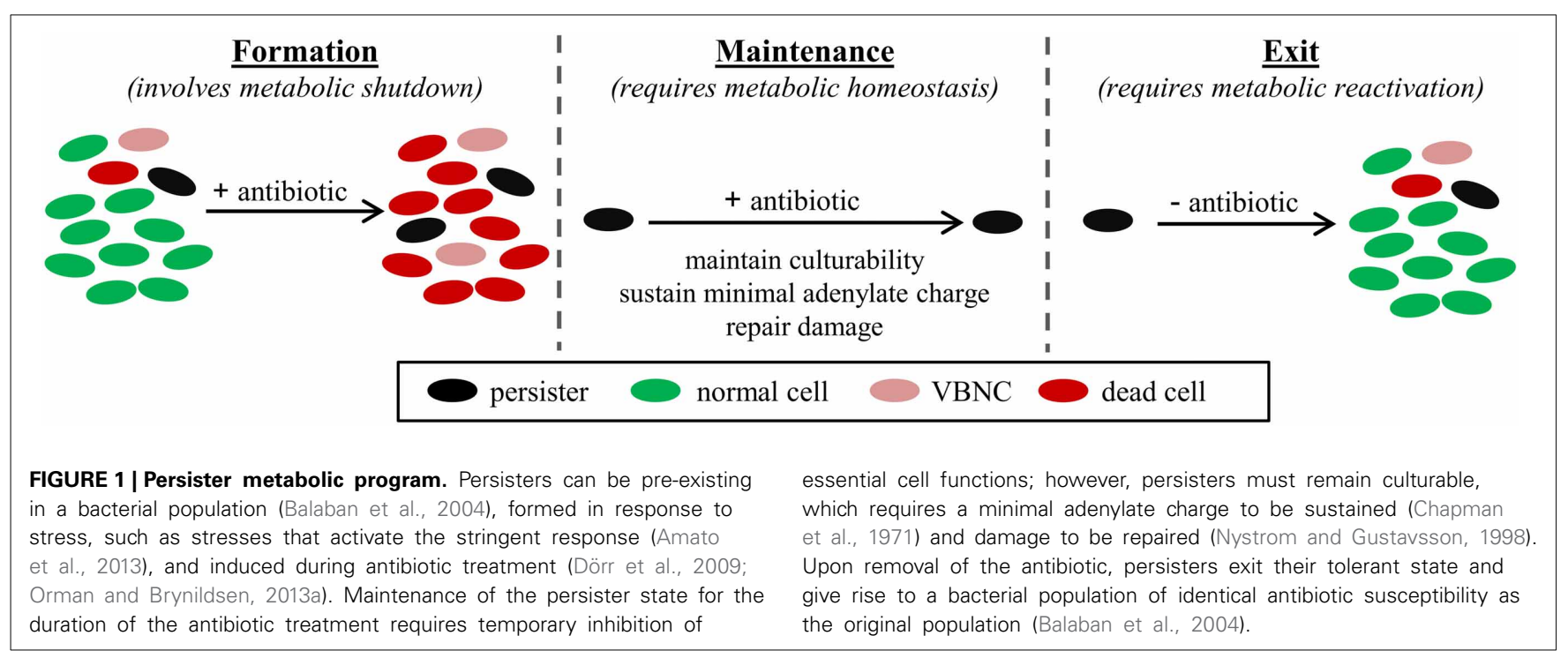

after successive rounds of selection on LB-AMP agar (Girgis et al., 2012). This effect was attributed to elevated levels of methylglyoxal, a toxic compound derived from DHAP. Interestingly, these observations, where GlpD inactivation increased persistence, were opposite to those of Spoering and colleagues. However, we note that G3P is a highly connected metabolite, given its proximity to central metabolism, interaction with the quinone pool, and use as a precursor for phospholipid biosynthesis. Therefore, different assay conditions may explain the variable impacts on persistence.

Beyond G3P, genomic studies have found that mutations perturbing amino acid (AA) metabolism significantly influence persistence (Table 1). Screening of an E. coli transposon library for persistence to ticarcillin (TIC) or OFL identified 18 mutants with increased persister levels, and of those, 16 mapped to genes involved in AA biosynthesis (Bernier et al., 2013). Pseudomonas aeruginosa screens have also uncovered disruptions in AA metabolism as important to persistence. Mutation of PA4115, a putative lysine decarboxylase, was found to increase persistence to carbenicillin (CB) (Manuel et al., 2010), whereas mutation of pheA, which is also involved in AA metabolism, was found to increase persistence to OFL (De Groote et al., 2009). These studies suggest that AA metabolism is a critical mediator of persistence, and as one would expect, the stringent response, a major metabolic regulatory system controlled by ppGpp and its transcriptional partner DksA, also mediates persistence (Korch et al., 2003; Viducic et al., 2006; Fung et al., 2010; Nguyen et al., 2011; Amato et al., 2013; Maisonneuve et al., 2013). This influence was also detected in a screen where $\Delta d k s A$ was found to produce far fewer persisters toward OFL (Hansen et al., 2008).

The third major metabolic system that has been shown to impact persistence is energy metabolism. A screen of an E. coli transposon library found that deactivation of $p h o U$ reduced persistence (Li and Zhang, 2007). PhoU is a negative regulator of the phosphate operon, and its inactivation led to a hyperactive metabolic state. In a screen of the Keio collection for AMP persistence, $\triangle s u c B$ and $\Delta u b i F$ were found to produce lower persister levels (Ma et al., 2010). SucB participates in the TCA cycle, whereas $\mathrm{UbiF}$ is an enzyme in ubiquinone biosynthesis, and deactivation of either of these genes leads to deficient energy production. Interestingly, these studies point to both metabolic hyperactivity and inhibition as methods to reduce persistence. One interpretation of these results could be that metabolic hyperactivity reduces entry into the persister state, whereas inhibition of energy production prevents exit from the phenotype. Regardless, energy generation appears to be a critical process to the persister metabolic program.

Collectively, these studies have provided a wealth of evidence on the importance of metabolism to bacterial persistence, even though they have sampled only a fraction of the mutational landscape. The details of how each genetic perturbation affects entry into, maintenance of, or exit from the persister state largely remains to be elucidated; however, it is clear that G3P, AA metabolism, and energy production are all important to defining persistence in a bacterial population.

\section{PERSISTER LEVELS DEPEND ON THE NUTRITIONAL ENVIRONMENT}

In addition to genetic evidence, the importance of metabolism to persistence has been supported by the impact of nutrient availability on persister levels. The most comprehensive investigation in this regard explored how the absence of AAs, glucose, ammonium, phosphate, and nucleobases altered persistence to AMP, OFL, and gentamicin (GEN) in E. coli (Fung et al., 2010). This study concluded that AA deprivation often increases persistence, mirroring the results from genomic screens that found mutations in AA metabolism to enhance persistence (Table 1). In a study of persister awakening, the number of E. coli persisters to AMP and norfloxacin (NOR) were found to be higher when the same stationary-phase culture was inoculated into media unable to support rapid growth resumption (minimal glycerol) in comparison to media with rapid regrowth (LB and minimal glucose) (Joers et al., 2010). Similarly, E. coli biofilms have been reported to exhibit higher tolerance to OFL or TIC in fresh media lacking 


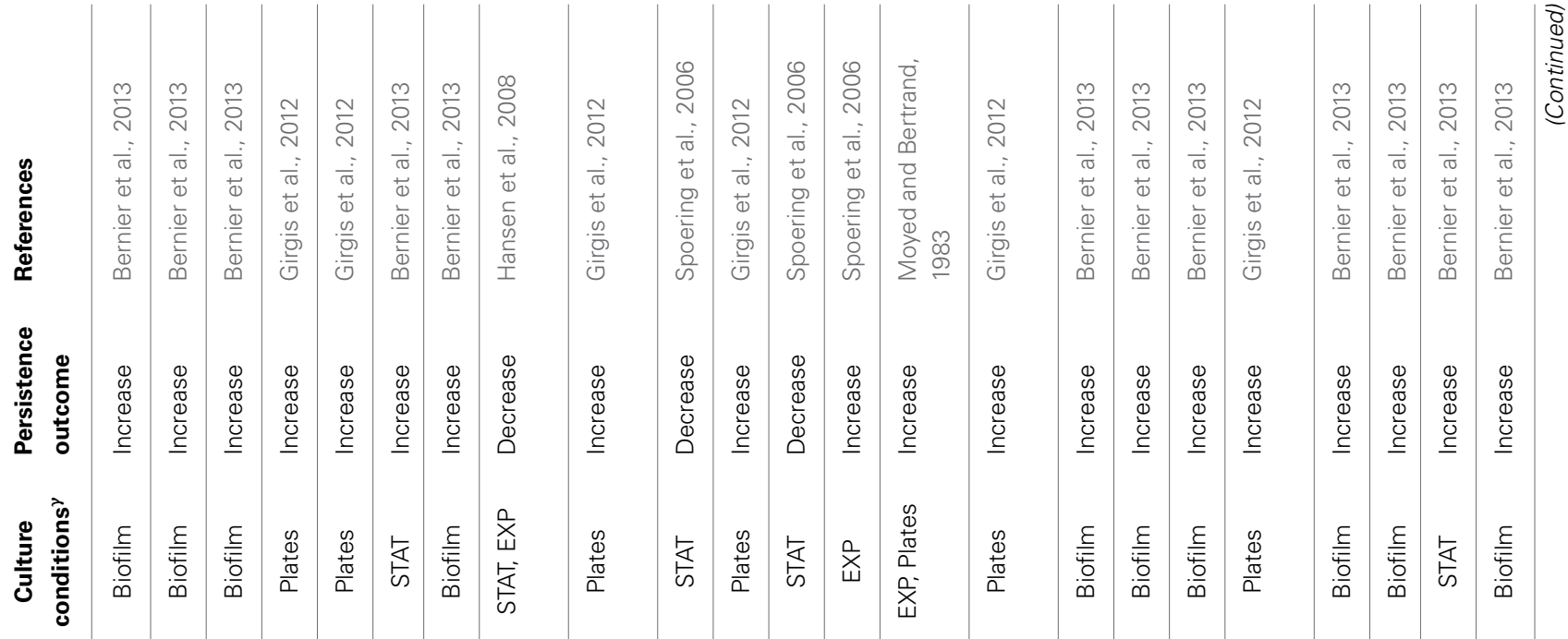

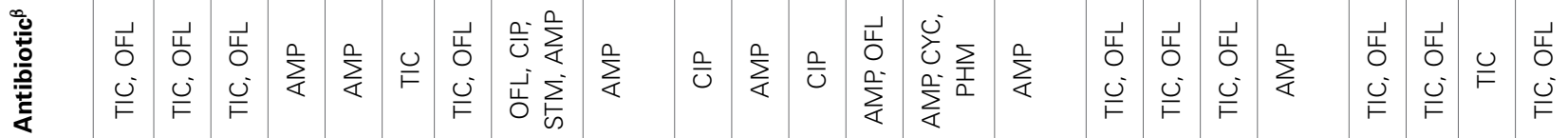
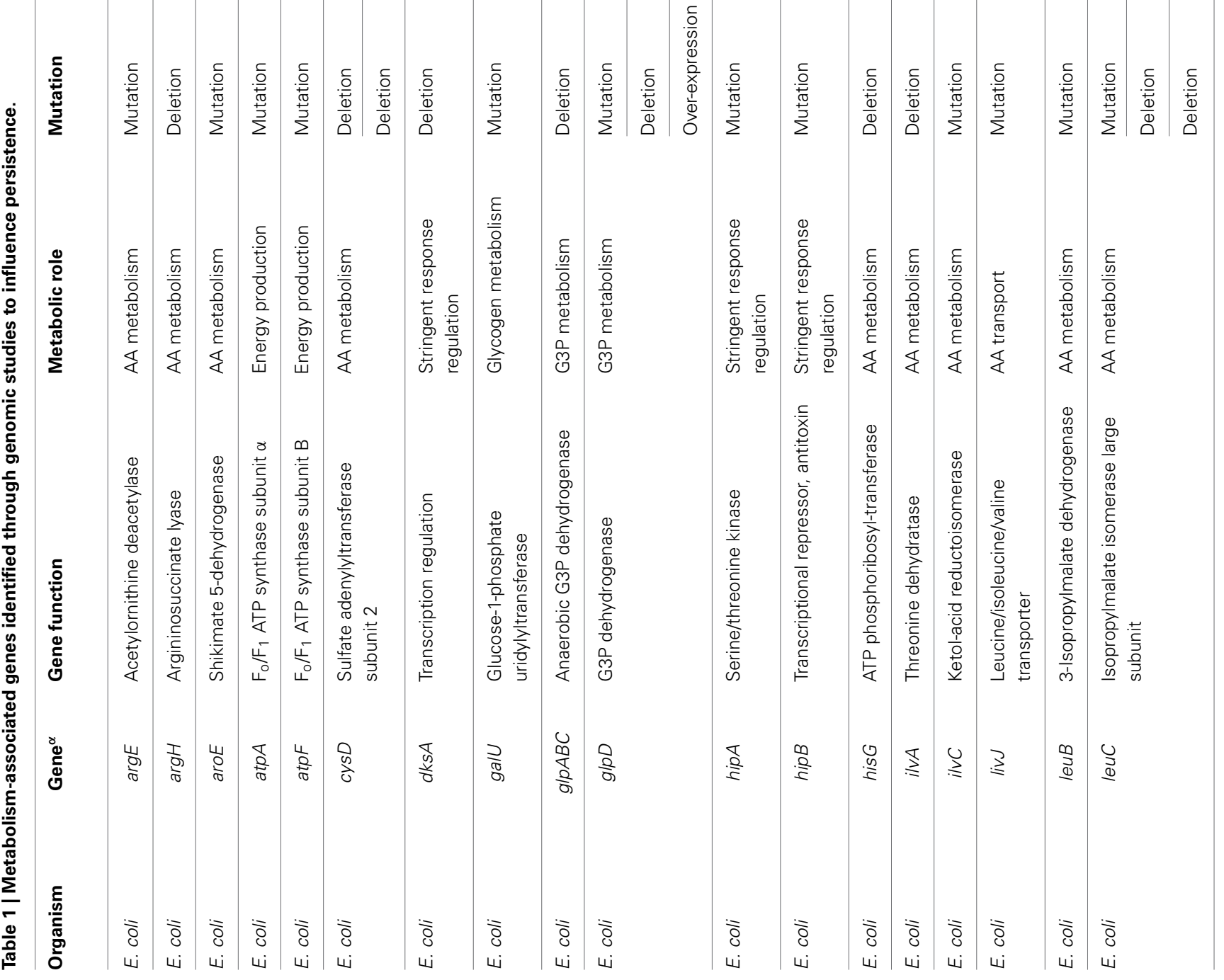


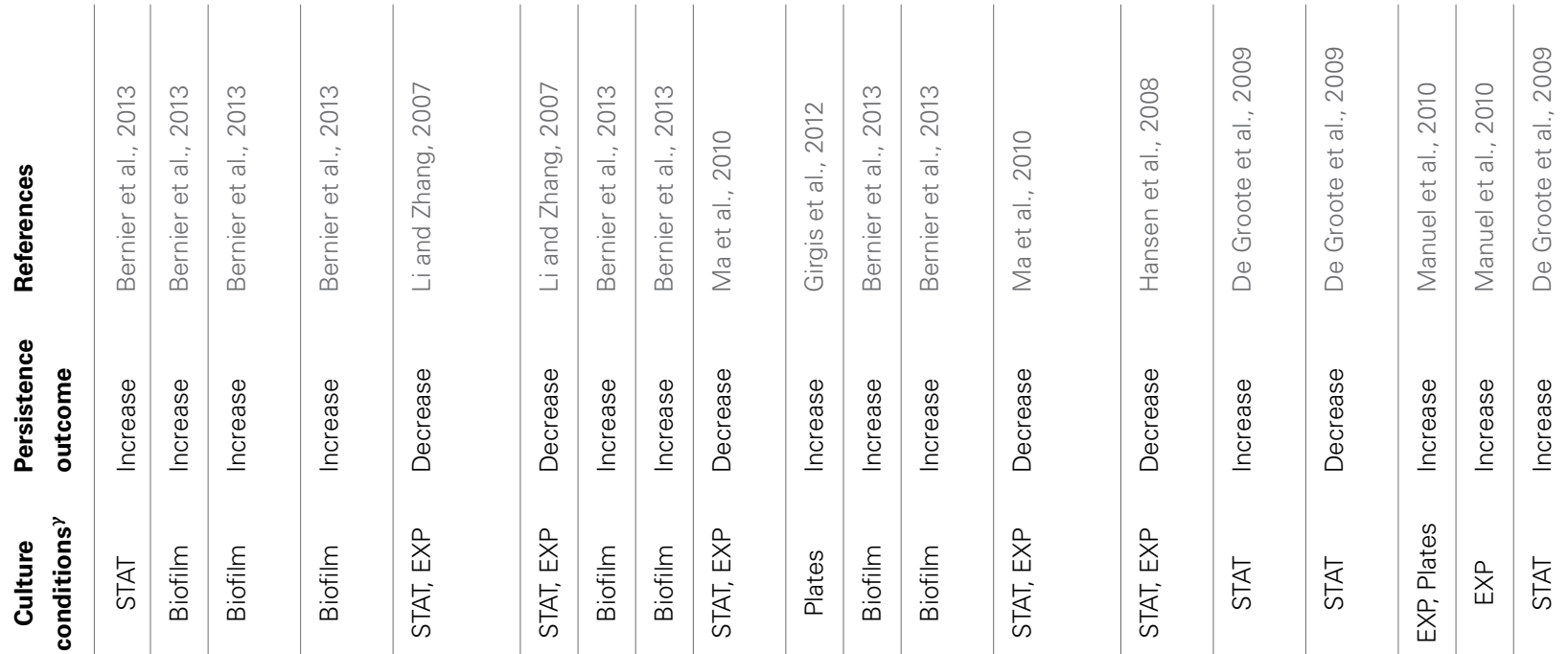

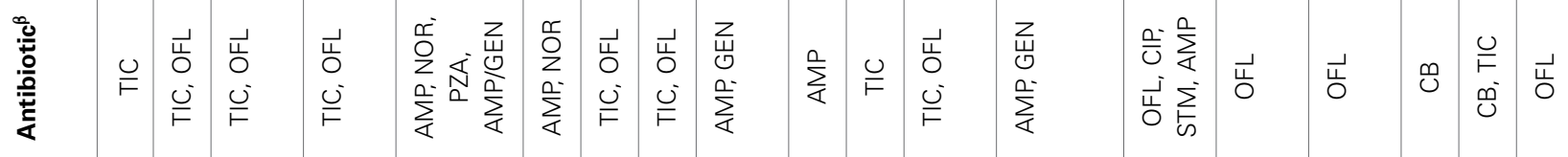

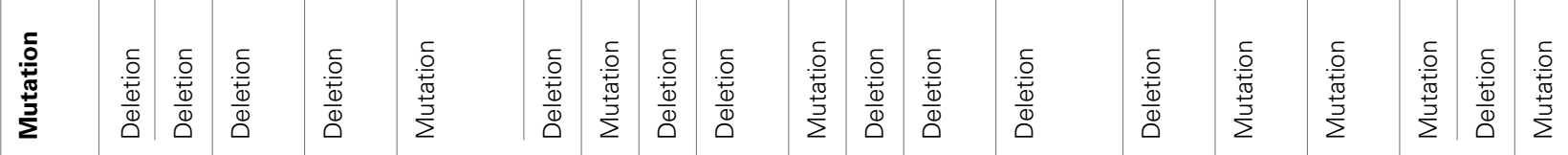
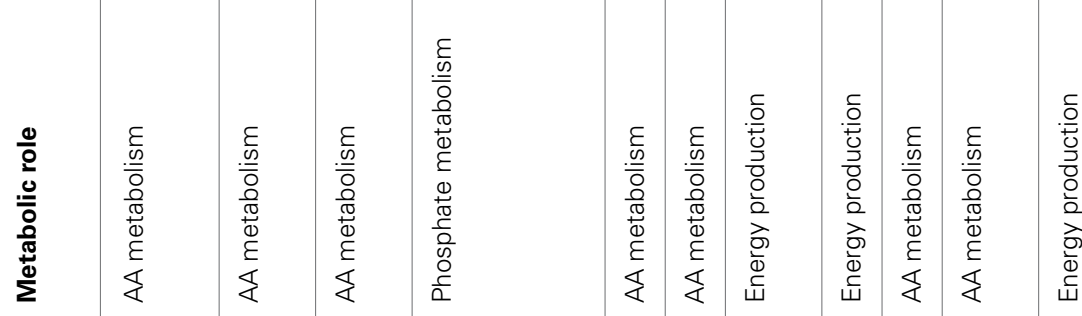

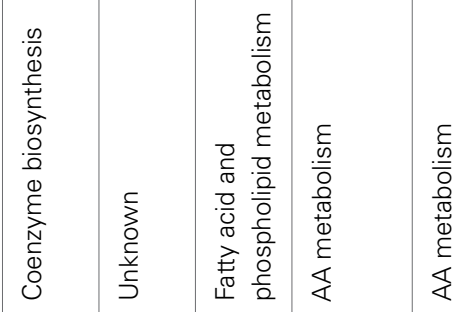
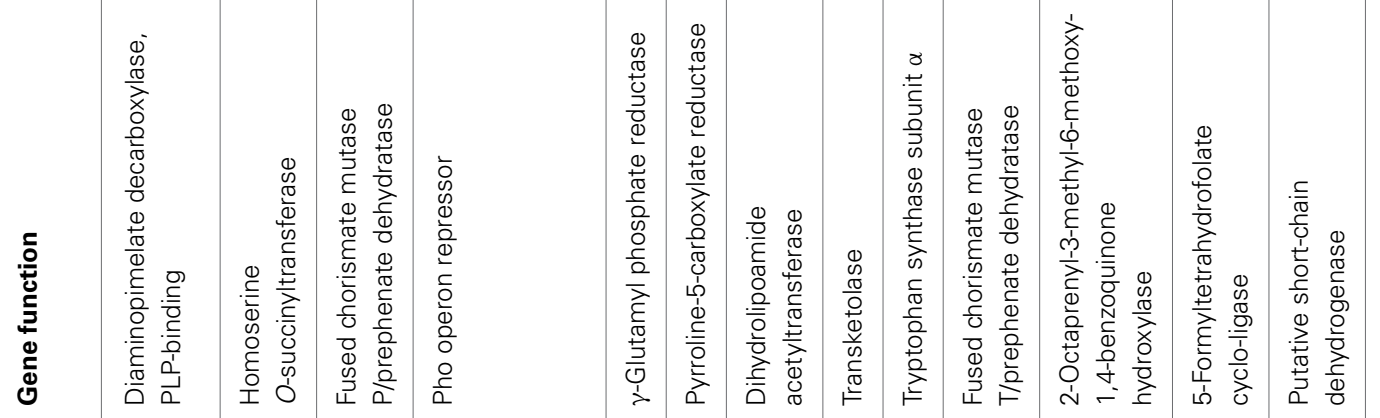

咅高

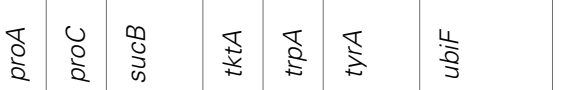

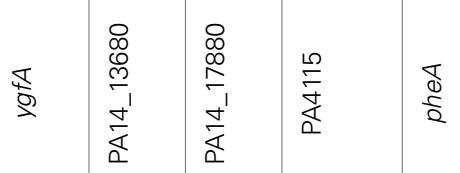

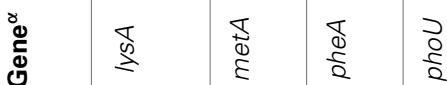
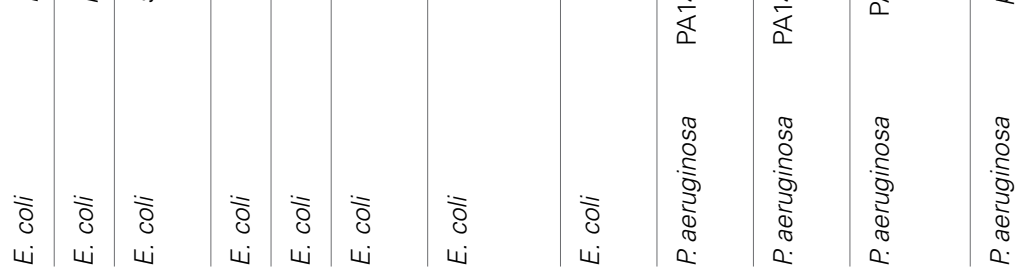


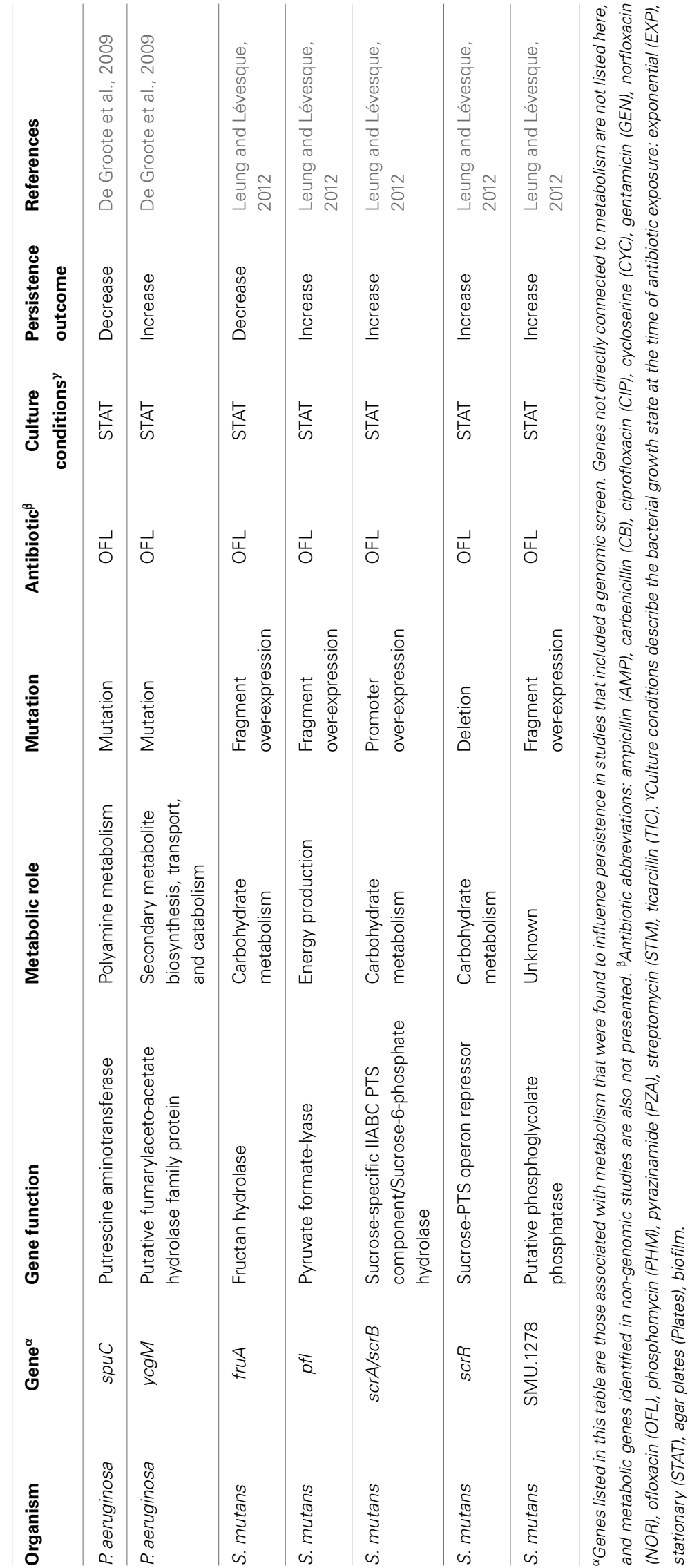


glucose, in comparison to controls with glucose (Bernier et al., 2013). Further support derives from the numerous studies that have shown that nutrient-limited stationary phase and biofilm cultures produce higher persister levels than their exponentially growing counterparts (Spoering and Lewis, 2001; Keren et al., 2004a; Lechner et al., 2012; Bernier et al., 2013). However, it is important to note that high density phenotypes such as quorum signaling may also contribute to persistence in such populations (Möker et al., 2010; Vega et al., 2012).

Taken together, these studies demonstrate that the nutritional environment directly influences persistence, suggesting a central role for metabolism in the persistence phenotype. Further, the mechanisms by which these nutritional stresses enhance persistence have been investigated, and ppGpp has been found to be a key mediator of this process.

\section{ppGpp, THE METABOLITE CONTROLLER OF PERSISTENCE}

ppGpp and the transcriptional regulator DksA are global regulators of metabolism (Traxler et al., 2006; Dalebroux and Swanson, 2012) that are critical mediators of persistence (Korch et al., 2003; Hansen et al., 2008; Amato et al., 2013; Bokinsky et al., 2013; Germain et al., 2013; Maisonneuve et al., 2013). In E. coli, AA limitation stimulates the ribosome-associated RelA to synthesize ppGpp, whereas various stress conditions, such as carbon (Xiao et al., 1991) and fatty acid starvation (Seyfzadeh et al., 1993), stimulate ppGpp synthesis from the cytoplasmic SpoT, which also encodes the sole ppGpp hydrolase. In conjunction with DksA, ppGpp interacts with RNA polymerase to inhibit transcription from stable ribosomal RNA promoters, while simultaneously upregulating transcription of AA biosynthesis and stress response genes (Potrykus and Cashel, 2008; Dalebroux and Swanson, 2012). ppGpp was initially associated with persistence through hipA7, a toxin mutant that required ppGpp for its elevated persister phenotype (Korch et al., 2003). Recent work on the native HipA has also shown that its impact on persistence requires ppGpp (Bokinsky et al., 2013; Germain et al., 2013). ppGpp can also increase persistence through its inhibition of exopolyphosphatase $(p p x)$, a modulator of the antitoxin degrading Lon protease (Maisonneuve et al., 2013). Additionally, we have demonstrated that RelA, SpoT, and DksA mediate persister formation in response to carbon source transitions (Amato et al., 2013). In particular, we found that the ppGpp biochemical network can act as a metabolic toxin-antitoxin module, where ppGpp is the metabolite toxin and SpoT is its enzymatic antitoxin. We demonstrated that increased ppGpp levels resulted in growth arrest and increased persistence, which could be reverted by SpoT coexpression, and using a mathematical model, we showed that the ppGpp biochemical network can exhibit bistability, where one subpopulation corresponds to normal cells (low ppGpp) and the other to persisters (high ppGpp). Interestingly, RelA-SpoT also demonstrate the prototypical conditional essentiality of a classical toxin-antitoxin system, where the toxin ( $\mathrm{relA}$ ) can be deleted, but the antitoxin (spoT) can only be removed in a strain without the toxin. In addition to $E$. coli, the stringent response has been shown to impact persistence in other organisms as well. In $P$. aeruginosa, RelA, SpoT, and DksA have all been found to impact persistence (Viducic et al., 2006; Nguyen et al., 2011), whereas in Mycobacterium tuberculosis, ppGpp was required for long term survival in an in vitro starvation and murine model (Primm et al., 2000; Dahl et al., 2003). Further, the mycobacterial stringent response was shown to exhibit bistability (Ghosh et al., 2011), supporting the assertion that ppGpp is a possible source of phenotypic heterogeneity. In addition, in Staphylococcus aureus, ppGpp has been shown to mediate antibiotic tolerance in response to cell envelope stress (Geiger et al., 2014).

These studies demonstrate the importance of the stringent response to persistence and highlight a prevalent mechanism by which metabolic stress can induce persistence. Considering this evidence supporting a central role for ppGpp in persistence, it is attractive to propose that an inhibitor of ppGpp synthesis, such as Relacin (Wexselblatt et al., 2012), or an activator of ppGpp hydrolysis could be effective therapeutics against persisters (Amato et al., 2013; Maisonneuve et al., 2013).

\section{PERSISTER METABOLISM AS A SOURCE OF ELIMINATION STRATEGIES}

To date, only a limited number of methods to kill persisters have been discovered, and interestingly, persister metabolism plays a vital role in each approach. For example, the first method, which we co-developed, used metabolites to stimulate proton motive force (pmf) generation in persisters, enabling aminoglycoside (AG) transport and their subsequent killing of E. coli and S. aureus persisters (Allison et al., 2011b). The participation of persister metabolism was confirmed with genetic mutants and chemical inhibitors, and subsequent studies have found the method to also be effective against $P$. aeruginosa persisters (Barraud et al., 2013). Another method was identified by Kim and colleagues, who screened a chemical library and found that a chemical named C10 promoted fluoroquinolone killing of E. coli persisters by stimulating their reversion to a replicating state (Kim et al., 2011). In another study, the quorum-sensing (QS) inhibitor BF8 facilitated elimination of $P$. aeruginosa persisters when combined with CIP or tobramycin (TOB) (Pan et al., 2012). However, upon further analysis, it was discovered that the effect of BF8 was likely due to reactivation of metabolism rather than inhibition of QS. Interestingly, BF8 has also been found to reduce E. coli persister levels when combined with OFL, tetracycline (TET), TOB, or GEN (Pan et al., 2013). Recently, another method to eliminate $S$. aureus persisters was discovered by leveraging knowledge that energy levels are low in persisters (Conlon et al., 2013). Specifically, ADEP4, which renders the ClpP protease ATPindependent, led to non-specific protein degradation and death in energy-depleted persisters. Taken together, these studies show that targeting persister metabolism holds great potential for the elimination of these dangerous bacteria and that greater knowledge of persister metabolism will facilitate the discovery of novel therapeutic strategies.

\section{METHODS TO MEASURE PERSISTER METABOLISM}

Given the potential of persister metabolism to yield anti-persister therapeutics, enhanced metabolic knowledge of these phenotypic variants is desirable. However, direct measurement of metabolites in persisters or assessment of their metabolic activities using conventional approaches, such as mass spectrometry and 
formazan-based colorimetric assays, are not currently possible due to isolation difficulties (Roostalu et al., 2008; Kint et al., 2012; Orman and Brynildsen, 2013a,b). Although several methods can provide persister-enriched samples (Keren et al., 2004b; Shah et al., 2006), such samples still contain many more other cell-types, such as normal cells and VBNCs, than persisters, and thus are of limited utility for metabolic measurements (Orman and Brynildsen, 2013b). Indeed, the major limitation to segregating persisters from a heterogeneous population is their similarity to VBNCs, which are often more highly abundant. Both VBNCs and persisters stain as live cells, harbor metabolic activity, and are non-growing under antibiotic stress. The only present difference between these subpopulations is that persisters resume growth on standard media, though we note that some VBNCs can regain culturability on non-standard media (Oliver, 2005) suggesting a role for the post-antibiotic environment in defining those cells that survive. Given these technical limitations, we have developed two methods to quantify persister metabolism. The first uses fluorescence activated cell sorting (FACS), a fluorescent measure of metabolic activity, and persistence assays to evaluate the metabolic status of persisters (Orman and Brynildsen, 2013a). This study, which provided the first direct measurement of persister metabolism, demonstrated that E. coli persisters largely contained low cellular reductase activity prior to antibiotic stress, confirming previous assumptions about the metabolic activity of the persister state. The second method leveraged the phenomenon by which specific metabolites enabled AG killing of persisters (Allison et al., 2011b). AG potentiation offered a rapid way to measure the breadth of persister metabolic activities (Orman and Brynildsen, 2013b), since the phenomenon relies on persister catabolism of nutrients for pmf generation. Persister metabolic activities are inferred from culturability on standard media, the distinguishing feature between VBNCs and persisters, thereby allowing investigation of persister metabolism even in the presence of VBNCs. This method enabled identification of nutrients metabolized by persisters to different antibiotics (AMP, OFL) during distinct growth stages (exponential, stationary), and thus allowed quantification of heterogeneity in persister metabolism. From these investigations, we demonstrated that glycerol and glucose are the most ubiquitously used carbon sources by various types of persisters, suggesting that the enzymes required for their catabolism are broadly available in persisters.

\section{CHALLENGES IN THE STUDY OF PERSISTER METABOLISM}

The technical hurdles associated with isolation of persisters have hindered understanding of the persister metabolic program and other aspects of persister physiology, including their transcriptome and proteome content. However, FACS offers a technical opportunity to discriminate between VBNC and persister phenotypes. For instance, mixed populations of VBNCs and persisters can be segregated from antibiotic-treated cultures using FACS (Roostalu et al., 2008; Orman and Brynildsen, 2013b), and since VBNCs are much more abundant than persisters in these samples, VBNC physiology can be quantified and potential biomarkers to discriminate between these two cell-types can be found. Nevertheless, any distinguishing features may be condition-specific, since numerous mechanisms can contribute to persister formation (Dhar and McKinney, 2007; Allison et al., 2011a; Balaban, 2011). Indeed, activation of particular pathways will depend on the environment and antibiotic used ( $\mathrm{Li}$ and Zhang, 2007; Luidalepp et al., 2011), and different formation mechanisms may be active in different growth stages, giving rise to persister heterogeneity, where multiple, distinct persister subpopulations, each with its own unique antibiotic tolerances, coexist in a bacterial culture (Allison et al., 2011a). As a result of heterogeneity, any isolation technique may only capture a fraction of the persisters present, yielding a limited sample of the persister population. Single-cell analysis techniques offer means to interrogate individual cells (Iino et al., 2012, 2013); however, the identification of persisters before they exit their non-replicative state is not presently possible. Perhaps a viable path forward is to study model persisters generated following the over-expression of genes that have been shown to increase persister levels (Korch and Hill, 2006; Vázquez-Laslop et al., 2006). Quantifying metabolic changes in these model systems may provide insight into the physiology and metabolic capabilities of different types of persisters (Bokinsky et al., 2013).

\section{CONCLUSION}

Persisters embody a medically important bacterial phenotype that relies on metabolism to establish and maintain a dormant, tolerant state during antibiotic stress, and exit that state upon removal of antibiotics (Figure 1). Considerable experimental evidence has accumulated substantiating the importance of metabolism to persistence, and the participation of metabolism in current persister eradication methods provides a convincing argument that enhanced knowledge of the persister metabolic program will accelerate the discovery of additional elimination strategies. However, isolation difficulties impede progress in the understanding of persister physiology, including metabolism. Two potential paths forward are to improve isolation techniques by studying the differences between persisters and VBNCs and to use model persisters to define the breadth and landscape of the persister metabolic program.

\section{ACKNOWLEDGMENTS}

Support was provided by the Department of the Army under award number W81XWH-12-2-0138 and the NIAID of the NIH under award number R21AI105342. The content is solely the responsibility of the authors and does not necessarily represent the official views of the funding agencies.

\section{REFERENCES}

Allison, K. R., Brynildsen, M. P., and Collins, J. J. (2011a). Heterogeneous bacterial persisters and engineering approaches to eliminate them. Curr. Opin. Microbiol. 14, 593-598. doi: 10.1016/j.mib.2011.09.002

Allison, K. R., Brynildsen, M. P., and Collins, J. J. (2011b). Metabolite-enabled eradication of bacterial persisters by aminoglycosides. Nature 473, 216-220. doi: 10.1038/nature10069

Amato, S. M., Orman, M. A., and Brynildsen, M. P. (2013). Metabolic control of persister formation in Escherichia coli. Mol. Cell 50, 475-487. doi: 10.1016/j.molcel.2013.04.002

Balaban, N. Q. (2011). Persistence: mechanisms for triggering and enhancing phenotypic variability. Curr. Opin. Genet. Dev. 21, 768-775. doi: 10.1016/j.gde.2011.10.001 
Balaban, N. Q., Merrin, J., Chait, R., Kowalik, L., and Leibler, S. (2004). Bacterial persistence as a phenotypic switch. Science 305, 1622-1625. doi: 10.1126/science. 1099390

Barraud, N., Buson, A., Jarolimek, W., and Rice, S. A. (2013). Mannitol enhances antibiotic sensitivity of persister bacteria in Pseudomonas aeruginosa biofilms. PLoS ONE 8:e84220. doi: 10.1371/journal.pone.0084220

Bernier, S. P., Lebeaux, D., Defrancesco, A. S., Valomon, A., Soubigou, G., Coppée, J.-Y., et al. (2013). Starvation, together with the SOS response, mediates high biofilm-specific tolerance to the fluoroquinolone ofloxacin. PLoS Genet. 9:e1003144. doi: 10.1371/journal.pgen.1003144

Bigger, J. W. (1944). The bactericidal action of penicillin on Staphylococcus pyogenes. Ir. J. Med. Sci. 19, 553-568. doi: 10.1007/BF02948386

Bokinsky, G., Baidoo, E. E., Akella, S., Burd, H., Weaver, D., Alonso-Gutierrez, J., et al. (2013). HipA-triggered growth arrest and $\beta$-lactam tolerance in Escherichia coli are mediated by RelA-dependent ppGpp synthesis. J. Bacteriol. 195, 3173-3182. doi: 10.1128/JB.02210-12

Chapman, A. G., Fall, L., and Atkinson, D. E. (1971). Adenylate energy charge in Escherichia coli during growth and starvation. J. Bacteriol. 108, 1072-1086.

Conlon, B. P., Nakayasu, E. S., Fleck, L. E., Lafleur, M. D., Isabella, V. M., Coleman, K., et al. (2013). Activated ClpP kills persisters and eradicates a chronic biofilm infection. Nature 503, 365-370. doi: 10.1038/nature12790

Dahl, J. L., Kraus, C. N., Boshoff, H. I. M., Doan, B., Foley, K., Avarbock, D., et al (2003). The role of RelMtb-mediated adaptation to stationary phase in longterm persistence of Mycobacterium tuberculosis in mice. Proc. Natl. Acad. Sci. U.S.A. 100, 10026-10031. doi: 10.1073/pnas.1631248100

Dalebroux, Z. D., and Swanson, M. S. (2012). ppGpp: magic beyond RNA polymerase. Nat. Rev. Microbiol. 10, 203-212. doi: 10.1038/nrmicro2720

De Groote, V. N., Verstraeten, N., Fauvart, M., Kint, C. I., Verbeeck, A. M., Beullens, S., et al. (2009). Novel persistence genes in Pseudomonas aeruginosa identified by high-throughput screening. FEMS Microbiol. Lett. 297, 73-79. doi: 10.1111/j.1574-6968.2009.01657.x

Dhar, N., and McKinney, J. D. (2007). Microbial phenotypic heterogeneity and antibiotic tolerance. Curr. Opin. Microbiol. 10, 30-38. doi: 10.1016/j.mib.2006.12.007

Dörr, T., Lewis, K., and Vulić, M. (2009). SOS response induces persistence to fluoroquinolones in Escherichia coli. PLoS Genet. 5:e1000760. doi: 10.1371/journal.pgen. 1000760

Fung, D. K. C., Chan, E. W. C., Chin, M. L., and Chan, R. C. Y. (2010). Delineation of a bacterial starvation stress response network which can mediate antibiotic tolerance development. Antimicrob. Agents Chemother. 54, 1082-1093. doi: 10.1128/AAC.01218-09

Geiger, T., Kastle, B., Gratani F., Goerke, C., and Wolz, C. (2014). Two small (p)ppGpp synthases in Staphylococcus aureus mediate tolerance against cell envelope stress conditions. J. Bacteriol. 196, 894-902. doi: 10.1128/JB.01201-13

Germain, E., Castro-Roa, D., Zenkin, N., and Gerdes, K. (2013). Molecular mechanism of bacterial persistence by HipA. Mol. Cell 52, 248-254. doi: 10.1016/j.molcel.2013.08.045

Ghosh, S., Sureka, K., Ghosh, B., Bose, I., Basu, J., and Kundu, M. (2011). Phenotypic heterogeneity in mycobacterial stringent response. BMC Syst. Biol. 5:18. doi: 10.1186/1752-0509-5-18

Girgis, H. S., Harris, K., and Tavazoie, S. (2012). Large mutational target size for rapid emergence of bacterial persistence. Proc. Natl. Acad. Sci. U.S.A. 109, 12740-12745. doi: 10.1073/pnas.1205124109

Hansen, S., Lewis, K., and Vulic, M. (2008). Role of global regulators and nucleotide metabolism in antibiotic tolerance in Escherichia coli. Antimicrob. Agents Chemother. 52, 2718-2726. doi: 10.1128/AAC.00144-08

Iino, R., Hayama, K., Amezawa, H., Sakakihara, S., Kim, S. H., Matsumono, Y., et al. (2012). A single-cell drug efflux assay in bacteria by using a directly accessible femtoliter droplet array. Lab Chip 12, 3923-3929. doi: 10.1039/c2lc40394c

Iino, R., Matsumoto, Y., Nishino, K., Yamaguchi, A., and Noji, H. (2013). Design of a large-scale femtoliter droplet array for single-cell analysis of drug-tolerant and drug-resistant bacteria. Front. Microbiol. 4:300. doi: 10.3389/fmicb.2013. 00300

Joers, A., Kaldalu, N., and Tenson, T. (2010). The frequency of persisters in Escherichia coli reflects the kinetics of awakening from dormancy. J. Bacteriol. 192, 3379-3384. doi: 10.1128/JB.00056-10

Keren, I., Kaldalu, N., Spoering, A., Wang, Y. P., and Lewis, K. (2004a). Persister cells and tolerance to antimicrobials. FEMS Microbiol. Lett. 230, 13-18. doi: 10.1016/S0378-1097(03)00856-5
Keren, I., Shah, D., Spoering, A., Kaldalu, N., and Lewis, K. (2004b). Specialized persister cells and the mechanism of multidrug tolerance in Escherichia coli. J. Bacteriol. 186, 8172-8180. doi: 10.1128/JB.186.24.8172-8180.2004

Kim, J. S., Heo, P., Yang, T. J., Lee, K. S., Cho, D. H., Kim, B. T., et al. (2011). Selective killing of bacterial persisters by a single chemical compound without affecting normal antibiotic-sensitive cells. Antimicrob. Agents Chemother. 55, 5380-5383. doi: 10.1128/AAC.00708-11

Kint, C. I., Verstraeten, N., Fauvart, M., and Michiels, J. (2012). New-found fundamentals of bacterial persistence. Trends Microbiol. 20, 577-585. doi: 10.1016/j.tim.2012.08.009

Korch, S. B., Henderson, T. A., and Hill, T. M. (2003). Characterization of the hipA7 allele of Escherichia coli and evidence that high persistence is governed by (p)ppGpp synthesis. Mol. Microbiol. 50, 1199-1213. doi: 10.1046/j.13652958.2003.03779.x

Korch, S. B., and Hill, T. M. (2006). Ectopic overexpression of wild-type and mutant hipA genes in Escherichia coli: effects on macromolecular synthesis and persister formation. J. Bacteriol. 188, 3826-3836. doi: 10.1128/JB.01740-05

Lechner, S., Lewis, K., and Bertram, R. (2012). Staphylococcus aureus persisters tolerant to bactericidal antibiotics. J. Mol. Microbiol. Biotechnol. 22, 235-244. doi: $10.1159 / 000342449$

Leung, V., and Lévesque, C. M. (2012). A stress-inducible quorum-sensing peptide mediates the formation of persister cells with noninherited multidrug tolerance. J. Bacteriol. 194, 2265-2274. doi: 10.1128/JB.06707-11

Lewis, K. (2008). Multidrug tolerance of biofilms and persister cells. Curr. Top. Microbiol. Immunol. 322, 107-131. doi: 10.1007/978-3-540-75418-3_6

Lewis, K. (2010). Persister Cells. Annu. Rev. Microbiol. 64, 357-372. doi: 10.1146/annurev.micro.112408.134306

Li, Y., and Zhang, Y. (2007). PhoU is a persistence switch involved in persister formation and tolerance to multiple antibiotics and stresses in Escherichia coli. Antimicrob. Agents Chemother. 51, 2092-2099. doi: 10.1128/AAC.00052-07

Luidalepp, H., Joers, A., Kaldalu, N., and Tenson, T. (2011). Age of inoculum strongly influences persister frequency and can mask effects of mutations implicated in altered persistence. J. Bacteriol. 193, 3598-3605. doi: 10.1128/JB. 00085-11

Ma, C., Sim, S., Shi, W., Du, L., Xing, D., and Zhang, Y. (2010). Energy production genes $s u c B$ and $u b i F$ are involved in persister survival and tolerance to multiple antibiotics and stresses in Escherichia coli. FEMS Microbiol. Lett. 303, 33-40. doi: 10.1111/j.1574-6968.2009.01857.x

Maisonneuve, E., Castro-Camargo, M., and Gerdes, K. (2013). (p)ppGpp controls bacterial persistence by stochastic induction of toxin-antitoxin activity. Cell 154, 1140-1150. doi: 10.1016/j.cell.2013.07.048

Manuel, J., Zhanel, G. G., and De Kievit, T. (2010). Cadaverine suppresses persistence to carboxypenicillins in Pseudomonas aeruginosa PAO1. Antimicrob. Agents Chemother. 54, 5173-5179. doi: 10.1128/AAC.01751-09

Möker, N., Dean, C. R., and Tao, J. (2010). Pseudomonas aeruginosa increases formation of multidrug-tolerant persister cells in response to quorum-sensing signaling molecules. J. Bacteriol. 192, 1946-1955. doi: 10.1128/JB.01231-09

Moyed, H. S., and Bertrand, K. P. (1983). hipA, a newly recognized gene of Escherichia coli $\mathrm{K}-12$ that affects frequency of persistence after inhibition of murein synthesis. J. Bacteriol. 155, 768-775.

Nguyen, D., Joshi-Datar, A., Lepine, F., Bauerle, E., Olakanmi, O., Beer, K., et al. (2011). Active starvation responses mediate antibiotic tolerance in biofilms and nutrient-limited bacteria. Science 334, 982-986. doi: 10.1126/science. 1211037

Nystrom, T., and Gustavsson, N. (1998). Maintenance energy requirement: what is required for stasis survival of Escherichia coli? Biochim. Biophys. Acta 1365, 225-231. doi: 10.1016/S0005-2728(98)00072-3

Oliver, J. D. (2005). The viable but nonculturable state in bacteria. J. Microbiol. 43, 93-100.

Orman, M. A., and Brynildsen, M. P. (2013a). Dormancy is not necessary or sufficient for bacterial persistence. Antimicrob. Agents Chemother. 57, 3230-3239. doi: 10.1128/AAC.00243-13

Orman, M. A., and Brynildsen, M. P. (2013b). Establishment of a method to rapidly assay bacterial persister metabolism. Antimicrob. Agents Chemother. 57, 4398-4409. doi: 10.1128/AAC.00372-13

Pan, J. C., Bahar, A. A., Syed, H., and Ren, D. C. (2012). Reverting antibiotic tolerance of Pseudomonas aeruginosa PAO1 persister cells by $(Z)-4$ bromo-5-(bromomethylene)-3-methylfuran-2(5H)-one. PLoS ONE 7:e45778. doi: 10.1371/journal.pone.0045778 
Pan, J. C., Xie, X., Tian, W., Bahar, A. A., Lin, N., Song, F. C., et al. (2013). (Z)-4Bromo-5-(bromomethylene)-3-methylfuran-2(5H)-one sensitizes Escherichia coli persister cells to antibiotics. Appl. Microbiol. Biotechnol. 97, 9145-9154. doi: 10.1007/s00253-013-5185-2

Potrykus, K., and Cashel, M. (2008). (p)ppGpp: still magical? Annu. Rev. Microbiol. 62, 35-51. doi: 10.1146/annurev.micro.62.081307.162903

Primm, T. P., Andersen, S. J., Mizrahi, V., Avarbock, D., Rubin, H., and Barry, C. E. 3rd. (2000). The stringent response of Mycobacterium tuberculosis is required for long-term survival. J. Bacteriol. 182, 4889-4898. doi: 10.1128/JB.182.17.48894898.2000

Roostalu, J., Jõers, A., Luidalepp, H., Kaldalu, N., and Tenson, T. (2008). Cell division in Escherichia coli cultures monitored at single cell resolution. BMC Microbiol. 8:68. doi: 10.1186/1471-2180-8-68

Seyfzadeh, M., Keener, J., and Nomura, M. (1993). spoT-dependent accumulation of guanosine tetraphosphate in response to fatty acid starvation in Escherichia coli. Proc. Natl. Acad. Sci. U.S.A. 90, 11004-11008. doi: 10.1073/pnas.90.23.11004

Shah, D., Zhang, Z. G., Khodursky, A., Kaldalu, N., Kurg, K., and Lewis, K. (2006). Persisters: a distinct physiological state of E. coli. BMC Microbiol. 6:53. doi: 10.1186/1471-2180-6-53

Spoering, A. L., and Lewis, K. (2001). Biofilms and planktonic cells of Pseudomonas aeruginosa have similar resistance to killing by antimicrobials. J. Bacteriol. 183, 6746-6751. doi: 10.1128/JB.183.23.6746-6751.2001

Spoering, A. L., Vulic, M., and Lewis, K. (2006). GlpD and PlsB participate in persister cell formation in Escherichia coli. J. Bacteriol. 188, 5136-5144. doi: 10.1128/JB.00369-06

Traxler, M. F., Chang, D.-E., and Conway, T. (2006). Guanosine $3^{\prime}, 5^{\prime}$ bispyrophosphate coordinates global gene expression during glucose-lactose diauxie in Escherichia coli. Proc. Natl. Acad. Sci. U.S.A. 103, 2374-2379. doi: 10.1073/pnas.0510995103

Vázquez-Laslop, N., Lee, H., and Neyfakh, A. A. (2006). Increased persistence in Escherichia coli caused by controlled expression of toxins or other unrelated proteins. J. Bacteriol. 188, 3494-3497. doi: 10.1128/JB.188.10.3494-3497.2006

Vega, N. M., Allison, K. R., Khalil, A. S., and Collins, J. J. (2012). Signaling-mediated bacterial persister formation. Nat. Chem. Biol. 8, 431-433. doi: 10.1038/nchembio. 915
Viducic, D., Ono, T., Murakami, K., Susilowati, H., Kayama, S., Hirota, K., et al. (2006). Functional analysis of spoT, relA and $d k s A$ genes on quinolone tolerance in Pseudomonas aeruginosa under nongrowing condition. Microbiol. Immunol. 50, 349-357. doi: 10.1111/j.1348-0421.2006. tb03793.x

Wakamoto, Y., Dhar, N., Chait, R., Schneider, K., Signorino-Gelo, F., Leibler, S., et al. (2013). Dynamic persistence of antibioticstressed mycobacteria. Science 339, 91-95. doi: 10.1126/science. 1229858

Wexselblatt, E., Oppenheimer-Shaanan, Y., Kaspy, I., London, N., SchuelerFurman, O., Yavin, E., et al. (2012). Relacin, a novel antibacterial agent targeting the stringent response. PLoS Pathog. 8:e1002925. doi: 10.1371/journal.ppat. 1002925

Xiao, H., Kalman, M., Ikehara, K., Zemel, S., Glaser, G., and Cashel, M. (1991). Residual guanosine $3^{\prime}, 5^{\prime}$-bispyrophosphate synthetic activity of relA null mutants can be eliminated by spoT null mutations. J. Biol. Chem. 266, 5980-5990.

Conflict of Interest Statement: The authors declare that the research was conducted in the absence of any commercial or financial relationships that could be construed as a potential conflict of interest.

Received: 21 December 2013; paper pending published: 13 January 2013; accepted: 09 February 2014; published online: 03 March 2014.

Citation: Amato SM, Fazen CH, Henry TC, Mok WWK, Orman MA, Sandvik EL, Volzing KG and Brynildsen MP (2014) The role of metabolism in bacterial persistence. Front. Microbiol. 5:70. doi: 10.3389/fmicb.2014.00070

This article was submitted to Microbial Physiology and Metabolism, a section of the journal Frontiers in Microbiology.

Copyright (C) 2014 Amato, Fazen, Henry, Mok, Orman, Sandvik, Volzing and Brynildsen. This is an open-access article distributed under the terms of the Creative Commons Attribution License (CC BY). The use, distribution or reproduction in other forums is permitted, provided the original author(s) or licensor are credited and that the original publication in this journal is cited, in accordance with accepted academic practice. No use, distribution or reproduction is permitted which does not comply with these terms. 